\title{
LA DIRECCIÓN DEL CAMBIO LINGÜÍSTICO. CLÍTICOS EN CORRIENTES Y EN BUENOS AIRES
}

\section{The direction of linguistic change. Clitics in Corrientes and Buenos Aires}

\author{
Angelita Martínez* \\ Universidad Nacional de La Plata \\ angemalucea@gmail.com
}

\author{
Palabras clave \\ variedades del español; \\ clíticos; \\ etnopragmática; \\ cambio lingüístico
}

\begin{abstract}
RESUMEN
En homenaje a la lingüista Inés Abadía de Quant y en reconocimiento a su dedicación al estudio de las variedades lingüísticas en contacto con el guaraní, en ese trabajo se contrasta el empleo de los pronombres clíticos de tercera persona lo, la y le en Corrientes y en Buenos Aires, como dos "centros de zonas dialectales diferenciadas" en la Argentina. A partir del concepto de juego intraparadigmático, se describen las estrategias comunicativas y etnopragmáticas que subyacen en el uso diferenciado de los clíticos. Se sostiene que, desde los mismos significados, se acude a explotaciones diferentes que cristalizan en distintas categorizaciones de la sustancia semántica. Evaluar la participación del actante en el evento como más activo o más afectado subyace la estrategia comunicativa. Los procesos cognitivos de activación del participante en el evento en Corrientes y de afectación del participante en Buenos Aires permiten una explicación etnopragmática y aportan al debate sobre la dirección del cambio.
\end{abstract}

\begin{abstract}
In honor of the linguist Inés Abadía de Quant and in recognition of her dedication to the study of linguistic varieties in contact with Guaraní, this paper contrasts the use of third-person clitic pronouns $l o, l a$ and $l e$ in Corrientes and in Buenos Aires, as two "centers of differentiated dialect zones" in Argentina. From the concept of "intraparadigmatic play", the communicative and ethnopragmatic strategies underlying the differentiated use of the clitics are described. It is argued that, from the same meanings, different exploitations crystallize in different categorizations of the semantic substance. Evaluating the participation of the actant in the event as more active or more affected underlies the communication strategy. The cognitive processes of activating the participant in the event in Corrientes and affecting the participant in Buenos Aires allow an ethnopragmatic explanation and contribute to the debate on the direction of change.
\end{abstract}




\section{Introducción}

Como todos sabemos, Inés Abadía de Quant ha investigado el español del litoral argentino en contacto con la lengua guaraní y se ha interesado, especialmente, por las diferencias dialectales respecto de otras áreas de nuestro país. En ese aspecto, a partir de un minucioso estudio de formas lexicales, ha caracterizado, acertadamente, a las ciudades de Corrientes y de Buenos Aires como dos centros dialectales diferentes (Abadía de Quant, 1996-1997; 2001). En cordial homenaje, hoy presentamos un contraste entre esas áreas lingüísticas, a partir de las diferencias que se observan en el empleo de los clíticos de tercera persona le y lo (la).

En efecto, en el ámbito de la gramática que estamos considerando, la región guaranítica argentina muestra una tendencia hacia el uso privilegiado de la forma le para señalar el acusativo mientras que, contrariamente, la región rioplatense ha privilegiado el empleo de lo/la y manifiesta un desplazamiento de dichas formas, incluso en contextos en los que habitualmente se seleccionaba la forma le.

Nuestra intención, específicamente, es mostrar que diferentes factores pueden influir en la selección de las formas y poner en evidencia la distinta explotación de los mismos significados. Este hecho nos recuerda que los parámetros que respaldan la selección no son universales (Martínez, 2000) y nos muestra, asimismo, que el rumbo de la variación y, eventualmente, la tendencia del cambio no es unidireccional (Martínez, 2019).

En lo que resta del artículo delinearemos y ejemplificaremos el problema de investigación; a continuación, haremos una pequeña síntesis de los principios teóricos en los que nos apoyamos, para luego exponer los factores diferenciales que influyen en el uso de los clíticos en ambos contextos geográficos y discursivos. Concluiremos con la interpretación de los resultados del análisis.

\section{El problema de investigación}

Como ya hemos dicho, en lo que respecta al empleo de los clíticos de tercera persona, se observan diferencias entre ambas zonas dialectales. Los ejemplos que siguen corresponden a emisiones recogidas en distintos medios gráficos editados actualmente en Corrientes.

(1) Estamos cansados de verle llorar a mi hermana! (Teleshow Paraguay, 12-7-2019)

(2) Karaí Octubre le llaman. Medio petisón es el hombre y su ancho sombrero lo achata aún más. (E1 Litoral, 1-10-2016)

(3) Se le abuyenta solamente con una olla repleta de comida. (El Litoral, 1-10-2016)

(4) Si no encuentra suficiente, se queda con esa familia para todo el año y, además de los rebencazos, la miseria les acompañará por todo el año, con sus nefastas consecuencias. (Corrientes chamamé, 1-10-2015)

(5) Un momento, no se apresuren! Les reprendió uno de sus jefes, antes debemos examinar si realmente este sujeto está muerto. (Corrientes chamamé, 27-12-2011) 
Las emisiones (1) a (5) ejemplifican el empleo de la forma le en situaciones de dos participantes en contextos en los que, en la variedad rioplatense, se selecciona, categóricamente, la forma lo(la), tal como en los ejemplos que siguen:

(6) El propio Melville se convenció al verla que podía confiarle, pese a su inexperiencia como actriz, el principal papel femenino de lo que resultó ser una obra maestra. (La Nación, 21-1-2021)

(7) La actual conquista deportiva de Marcelo Bielsa cobra un realce enorme en virtud de los valores que este entrenador sabe poner en juego. Por lo pronto, vale preguntarse: ¿por qué lo llaman loco? (Página 12, 22-7-2020)

(8) Si se lo abuyenta, se aleja a corta distancia volando bajo antes de esconderse entre la maleza. (https:// www.audubon.org/es/guia-de-aves/ave/chingolo-de-henslow)

(9) Lo acompaña desde hace algo más de dos años, desde que empezó su trabajo en Dorados, en México. (La Nación, 26-11-202)

(10) En aquel momento, las autoridades la reprendieron por haber informado sobre el tema. (La Nación, 1-4-2020)

A su vez, es sabido que, en Buenos Aires, otros verbos constituyen contextos variables. Entre ellos, le/lo preocupa, le/lo molesta, le/lo ayuda. Esta tendencia hacia el empleo del acusativo se va instalando actualmente, incluso, en situaciones de tres participantes. La variación se registra, en efecto, como un cambio incipiente, con algunos verbos -robar, aconsejar, informar, exigir- en contextos determinados.

Nuestro propósito es contrastar los dos escenarios aludidos a partir de un análisis que pone en evidencia el juego intraparadigmático como respuesta a perfilamientos cognitivos diferentes frente a la transitividad.

\section{Marco teórico}

Nuestro interés por el estudio de las variedades de una lengua nos ha llevado a desarrollar el concepto de juego intraparadigmático (Martínez, 2012, 2019) ${ }^{1}$ a partir de la observación de que en las fronteras intracategoriales del paradigma se producen desplazamientos, cuya causa está ligada al punto de vista desde el cual se está categorizando, es decir, desde el cual se reparte la sustancia semántica. Muchas veces, dicho punto de vista se relaciona con valores culturales de una comunidad y, entonces, el analista puede hallar explicaciones etnopragmáticas.

En general, los resultados de nuestras investigaciones nos han permitido reconocer que las diferencias entre variedades se corresponden con dichos desplazamientos intracategoriales. Por ello, consideramos fundamental emprender el análisis desde la perspectiva de la variación lingüística, que

1. Usamos la palabra "juego" en el sentido de la definición que presenta el Diccionario de la lengua española de la Real Academia Española, en la acepción número 6. "Disposición con que están unidas dos cosas, de suerte que sin separarse, puedan tener movimiento, como las coyunturas, los goznes, etc.” (Martínez, 2019, p. 200). 
entendemos como la selección de formas referencialmente equivalentes pero que aportan diferente significado. La selección se lleva a cabo de acuerdo con la perspectiva cognitiva con la que el emisor conceptualiza una escena y desea transmitir el mensaje (García, 1985, 1992).

En esta búsqueda, postular los significados de las formas, como categorías lingüísticas de una sustancia semántica particular, es esencial al análisis. Por su parte, la explotación de esos significados y su coherencia contextual nos permiten descubrir las estrategias comunicativas que subyacen al mensaje. La comprensión de dichas estrategias nos posibilita evaluar, una y otra vez, la hipótesis sobre el signficado de las formas lingüísticas en cuestión.

El análisis etnopragmático constituye, así, un desafío para la comprensión de la conformación de las gramáticas, entendidas como emergentes (Hopper, 1987) y cuya sintaxis está semántica y pragmáticamente motivada. Cuando las correlaciones gramaticales de dos zonas o comunidades se manifiestan diferentes, podemos sospechar que están en juego distintas categorizaciones sistemáticas. Al decir de García: "El descubrimiento y la interpretación de las correlaciones exige, por supuesto, un análisis del contenido semántico de las formas y sensibilidad para el valor de diversos contextos" (1995, p. 70).

\section{Empleo de los clíticos en Corrientes}

En lo que respecta a nuestro trabajo sobre el empleo de los clíticos (Martínez, 1996, 2000, 2008, 2010), hemos intentado mostrar que, contrariamente a la versión tradicional que hace referencia a "focos de leísmo en América" (Vidal de Battini, 1964, p. 61-62), el uso frecuente de la forma le para remitir acusativos, propio de la región guaranítica argentina, no se corresponde con la estrategia desarrollada en Castilla. Si bien superficialmente pareciera tratarse del mismo fenómeno lingüístico, la diferencia resulta medular puesto que, mientras que el paradigma innovador castellano ha sido descrito como la recategorización de la sustancia de caso en favor de la de género (García, 1986, 1992; Klein-Andreu, 1999), en la región del litoral argentino, el valor de los clíticos le, lo y la sigue siendo el etimológico de caso, sin excepción.

En dicha variedad, la ausencia de laísmo y el empleo de la forma le para señalar referentes femeninos tanto como masculinos constituyen pruebas cruciales para la falsación de la hipótesis que asimila el uso del litoral al leísmo peninsular en que la categorización de género (le para el masculino; la para el femenino y lo para lo no discreto) ha reemplazado a la categorización de participación del actante en el evento, o sea, al caso.

Por otra parte, los estudios llevados a cabo a partir de diversos corpus de Corrientes (Martínez, 1996, 2000; Gonzalez Sandoval, 2005; Yausaz, 2005) coinciden en destacar la explotación del dativo como evaluación de mayor actividad relativa del actante en el evento. ${ }^{2}$

2. Este uso extendido del pronombre le puede estar influido por el contacto del español con el guaraní, dadas las características de esta lengua en el ámbito de la referencia a la tercera persona (Abadía de Quant, 1996; Abadía de Quant e Yrigoyen, 1980; Granda, 1982; Palacios, 2008; Martínez, 2000; González, 2005). En efecto, algunos rasgos de la gramática guaraní que podrían estimularlo son: a) La ausencia de marca morfológica de género. b) La presencia de la posposición pe -y sus alomorfos -ve, -me $\approx-$ para señalar al objeto animado. 
En efecto, el análisis de los datos recogidos por Vidal de Battini (1984), en leyendas narradas en Corrientes, muestra que son factores ligados al grado de actividad otorgado al referente los que influyen en la selección de las formas. Dadas las características del género leyenda, no es de extrañar que los participantes connotados culturalmente (Martínez, 1996, 2000), que son respetados, temidos o venerados, sean los referentes favorecidos por la frecuencia con que se los remite a través de la forma le. En este contexto, seguramente, dada la relevancia de la presencia de la Virgen de Itatí como entidad culturalmente connotada, los femeninos referidos mediante le muestran una frecuencia relativa mayor. Por ejemplo:

(11) La Virgen de Itatí é muy milagrosa. La Virgen jue hallada por milagro también. Dice que un indio le encontró en una islita, en Itatí mismo, en el río Paraná. Dice que hizo milagro. Dice que le venía a vé gente de todo lado y a todo le hacía el milagro de toda clase. Ya le conocía en toda parte.

Dice que le trajo a Corriente, que le quería tener en la Capital. Esa noche misma la Virgen se jue a Itatí. Dice que le trajo do vece más y la Virgen se volvía siempre. Ya vieron que se quería quedá en Itatí. En Itatí le hicieron capilla por ella, una linda capilla. Dice que la Virgen tenía como música, que era como una música del cielo, que se solía oír cuando entraba a la capilla.

Dice que se quedó Nuestra Señora en Itatí, y entonce se formó el pueblo de Itatí con esta Patrona. Dice que ella lo defendía de lo indio. Despué dice que lo defendió de lo paraguayo, en la guerra del Paraguay. (VIII: 180-181)

Como vemos en (11), el narrador alterna las formas le y lo. Cuando remite a la virgen selecciona el dativo le, coherentemente con la evaluación de actividad del personaje connotado, más allá del género del mismo. Contrariamente, mediante la selección de lo remite a un referente más neutral, en este caso, al pueblo de Itatí.

Coherentemente, en el ejemplo siguiente, mostramos que también otros seres respetados o temidos, como el mítico Talonyovai, son referidos vía le:

(12) A mí me solían decir que el talónyovái era un indiecito. Que tenía en lo pie do talón. Que no tenía dedo por lo pie. Si uno le quería seguir no sabía para dónde va, porque el lado que uno miraba, la pisada tenía talón. É chiquito, pero muy malo y tiene mucha fuerza.

Talónyovái quiere decir talón por frente de otro talón. Me solían decir que era muy malo, que si encontraba a la criatura la comía. Yo le tenía mucho miedo al talónyovái. Ya se ve poco. Sólo en el monte, lejo, se puede encontrar el talónyovái. (VIII, 2230, 680)

Como en (11), una vez más la variación en el uso de los clíticos da cuenta de la estrategia comunicativa del narrador: asignar un plus de actividad al referente connotado. Como vemos, la forma del dativo se utiliza para remitir al ser extraordinario mientras que la referencia a una criatura habitual se lleva a cabo a través de la forma acusativa la.

El corpus que nos aporta González Sandoval (2005) registra producciones escritas de estudiantes goyanos y nos ha permitido comprobar (Martínez, 2010, 2013) que, en consonancia con los datos que obtuvimos en las leyendas y cuentos populares de Vidal de Battini, la alternancia de los clíticos le, lo/la, en situaciones de dos participantes, se ve favorecida por la misma necesidad comunicativa de señalar 
referentes de acuerdo con el grado de actividad otorgada por el enunciador. En este caso, la variación hallada en los registros de los estudiantes se relaciona con la animacidad del referente, contrastando, específicamente, la vida y la muerte. ${ }^{3}$ En ese aspecto, los ejemplos son concluyentes, tal como podemos apreciar en (13) en que la variación en la selección del clítico da cuenta de la estrategia comunicativa subyacente:

(13) Cuando Marisol se paró para cambiar la yerba del mate, a la pobre le picó una víbora ponzoñosa. $\mathrm{Al}$ instante le llevamos al hospital, pero ella no pudo aguantar tanto tiempo y murió.

Hoy en día, cuando salimos todos juntos, la recordamos y la extrañamos mucho. (Goya, 37, 182)

La misma estrategia comunicativa se visualiza, incluso, cuando se trata de una muerte aparente:

(14) Cuando mi hermano se iba subiendo vimos una víbora que era una yarará amarilla. Mi hermano se asustó porque casi le muerde. Yo me bajé rápido y le pegué con un palo en la cabeza. Mi hermano me dijo. "Ya está muerta". Yo le dije "Sí"; agarramos una piola y la llevamos a la casa de mi tía. Cuando estábamos pasando la laguna, la víbora se movió y salió de la piola, se escondió detrás de los camalotes, y cuando volvimos a la vuelta le vimos comiendo un pescado. (Goya, 75, 189)

La sutileza en el aprovechamiento del significado de las formas y el grado de abstracción que subyace a la selección de las mismas permite apreciar, una y otra vez, el talento humano (Diver, 2012), en su necesidad de comunicar algo que considera relevante.

En esta instancia, nos resulta necesario señalar la influencia del género discursivo en el empleo variable de las formas. En efecto, tanto en las leyendas como en las producciones escritas de los escolares se lleva a cabo la explotación de los mismos significados relacionados con el grado de actividad del actante en el evento, pero a la luz de las características de los referentes del discurso: seres connotados y neutros en las leyendas, seres cotidianamente enfrentados al contraste de la vida y de la muerte en las producciones discursivas de los niños. Una prueba más de que la evaluación del referente en lo que corresponde al grado de actividad relativa asignada es relevante para los usuarios de la lengua.

Yausaz (2005), por su parte, a partir de textos orales y escritos por estudiantes de tercer año de una escuela de Corrientes, enfatiza la diferencia entre la oralidad y la escritura respecto del empleo de le, en situaciones de dos participantes. La influencia de la normativa escolar y el contacto constante con los medios de comunicación provenientes de Buenos Aires son, para el autor, los promotores de que, en la escritura, se instale el empleo rioplatense.

Pero incluso ante esa realidad se siguen registrando casos en la prensa escrita, tal como los consignados más arriba, que atienden a la necesidad comunicativa de empleo del dativo le, propia de la región.

3. También Yausaz (2005) analiza este corpus y considera que la forma le se emplea para distinguir referentes animados. De la misma manera, los análisis realizados en contexto de otras provincias de la región, como Chaco (Martínez, 2000) o Misiones, son consistentes con la hipótesis de grado de actividad del referente. En lo que respecta a Misiones, la distribución de las estrategias le(s) y cero se encuentra determinada, más que nada, por factores lingüísticos, con mayor énfasis en la animacidad del referente (De Ramos, 2017). 


\section{La región rioplatense argentina}

Contrariamente, las investigaciones del empleo de clíticos en la región rioplatense que hemos llevado a cabo (Martínez, 2008, 2010, 2019) nos permiten comprobar una tendencia al aumento de frecuencia de selección de lo en contextos en los que esperábamos, habitualmente, la forma le.

Como ya hemos dicho, en algunos contextos verbales -como (1) a (5) - el empleo de la forma del dativo en situaciones de dos participantes resulta ajeno a la variedad rioplatense.

En cuanto a los usos variables, hemos estudiado la alternancia de las formas lo y le, en Buenos Aires, con los verbos: preocupar (Martínez, 2008), molestar (Martínez, 2013), ayudar (Mauder y Martínez, 2019). En dichos contextos se pone en evidencia un notable avance de las formas lo/la preocupa, lo/la molesta, lo/la ayuda, en relación con las construcciones más conservadoras: le preocupa, le molesta, le ayuda.

Esta necesidad de expresar la afectación del agente sobre el objeto se manifiesta, también, en las creaciones: lo desaparecieron, lo suicidaron, lo fueron, que son, desde hace unos años, expresiones de empleo habitual en el español rioplatense.

En efecto, establecer un nivel mayor de afectación del referente del clítico se manifiesta como una necesidad comunicativa, en algunos eventos de habla, entre los hablantes de Buenos Aires. Cuando se desea asignar un grado de afectación mayor que el inferido por el uso canónico, se recurre, coherentemente con su aporte significativo lo menos activo, al clítico lo.

Pero lo más sorprendente es que la variedad rioplatense argentina avanza, también, hacia el empleo de la forma $l o$ incluso en algunas situaciones de tres participantes. Es el caso de expresiones con el verbo robar, como la que sigue: ${ }^{4}$

(15) No tienen perdón de Dios. Ataron a sacerdote para robarlo.(Crónica, 21-12-2020)

De acuerdo con nuestra investigación en lo que respecta al verbo robar (Martínez 2019), lo que se observa actualmente en Buenos Aires, cuando el clítico remite al afectado y la entidad robada no se halla explicitada, es un empleo innovador en el que comienza a alternar la forma le (a X le robaron) con las formas lolla (a X lo robaron), en posición tanto antepuesta como pospuesta.

En efecto, hemos observado que en dichas emisiones la variación en el empleo del clítico lel lo(la) se halla ligada a la necesidad de señalar a la víctima como un referente directa o indirectamente afectado por el evento. La forma lo (la) con su significado lo menos activo favorecerá el discurso orientado directamente al afectado (Martínez, 2019).

El análisis de nuestros datos revela, además, que la violencia física en la escena narrada es un factor que favorece la presencia de lo. Esto es consistente con la necesidad de señalar a una víctima muy

4. Nuestro corpus revela que la variación está activa y que la misma ha ido en aumento desde 1996 a 2015 . En efecto, en el periódico considerado, se registra, en 1996, una única mención de lo + verbo robar en situaciones de tres participantes como las estudiadas mientras que, en 2014, se registran doce menciones. 
afectada mediante el clítico que expresa lo menos activo del evento. Los ejemplos que siguen (Martínez, 2019) dan cuenta de esta estrategia:

(16) Subió a un taxi con una impresora y lo robaron. Le dieron un culatazo en el ojo que casi le hace perder la visión. $(6 / 11 / 2001)$

(17) Abrió el negocio hace tres meses y ya lo robaron a mano armada en cuatro ocasiones. En dos de ellas lo golpearon, llevándose dinero y mercancías. (16/03/1998)

\section{Activación vs. afectación del referente}

Como hemos observado, si contrastamos ambas áreas dialectales, en el español correntino, la estrategia de los hablantes y escribientes tiene que ver con lo que vamos a llamar la activación del referente. En situaciones de dos participantes, dos actantes se hallan polarizados en lo que respecta a su grado de actividad o de participación en el evento: el sujeto y el objeto. El sujeto es el actante más activo del evento y, como contraparte, el objeto resulta lo menos activo del evento. Pero ese objeto puede ser categorizado, relativamente, menos activo (y no lo menos activo) y entonces se lo remite mediante la forma le. Así, la forma y el significado del dativo invade el terreno de los acusativos, en situaciones de dos participantes, para señalar que el referente es evaluado menos polarizado, más cercano al sujeto en grado de actividad. Ya no es conceptualizado como lo menos activo del evento sino solo como menos activo que el sujeto. De ahí que el empleo de le en Corrientes, como hemos mostrado, favorezca referentes humanos, seres vivos o, en ciertos contextos, entidades culturalmente connotadas.

En contraste, en Buenos Aires, la estrategia que prospera es la que vamos a llamar afectación del referente. Y el procedimiento es el contrario. Se trata de desactivar al objeto, presentarlo más afectado. Consistentemente, entonces, se recurre a la forma lo para referir al actante menos activo y presentarlo como lo menos activo del evento. La misma estrategia vale para situaciones de dos participantes (lo preocupa la situación del país) como de tres participantes (lo robaron cuando iba a su trabajo).

Como vemos, el dinamismo lingüístico y, de ahí, la configuración permanente de las gramáticas, permanecen activos. El juego intra-paradigmático nos ofrece, a los usuarios del intrumento comunicativo, esa gran posibilidad.

\section{Conclusiones}

El empleo de los clíticos en la Argentina presenta un área de flexibilidad entre el acusativo y el dativo en permanente movimiento. E1 hablante o escribiente, ante la necesidad de manifestar el grado de actividad que asigna al referente, selecciona la forma más adecuada a su evaluación.

El empleo observado en las dos regiones contrastadas indica que se trata de una misma estrategia comunicativa: evaluar el grado de actividad de los actantes en evento. La diferencia radica, como hemos tratado de mostrar, en el perfilamiento etnopragmático desde el cual se transmite el mensaje. 
En la región guaranítica, específicamente, en el corpus correspondiente a Corrientes, el empleo de la forma del dativo, le, se expande e invade al acusativo cuando el emisor señala referentes considerados más activos: animados, humanos, connotados culturalmente, vivos frente a muertos.

Contrariamente, en el ámbito rioplatense, el clítico acusativo lo invade al dativo para señalar una preocupación social al poner en evidencia la percepción de inseguridad ante el aumento de la violencia en los delitos callejeros.

En lo que respecta al impacto teórico de esta confrontación de dos variedades del español de la Argentina, el trabajo nos permite apoyar la idea de que los aspectos culturales de una región influyen en la estructuración de la lengua. Los factores que inciden en el empleo de las formas responden a los perfilamientos comunicativos de los hablantes y se manifiestan, sistemáticamente, en el juego intraparadigmático. La explotación de dichos significados es producto de la creatividad infinita de los hablantes y va delineando la identidad comunitaria.

Y esto constituye un llamado de atención al hecho de que hablar de corrección lingüística resulta inadecuado.

Por otra parte, insistimos en que la frecuencia de empleo de le en Corrientes no es la consecuencia de una recategorización del sistema como la manifestada en el norte de España. No hay leísmo ni loísmo a la manera peninsular. Hay una explotación diferente de los significados etimológicos que continúa privilegiando un sistema de caso (grado de actividad del actante en el evento) y que, solo marginalmente, señala el género.

Asismismo, el contraste en la explotación de los mismos significados en las dos variedades nos abre camino hacia otro aspecto que impacta en la teoría lingüística general: la direccionalidad del cambio lingüístico. La variedad rioplatense muestra una tendencia inversa al resto de América en donde, según los estudios llevados a cabo en los últimos años, se observa un aumento de la selección del dativo sobre el acusativo. La tendencia rioplatense al desplazamiento del acusativo sobre el dativo es una realidad que pone en discusión la unidireccionalidad del cambio que es auspiciada, aún, en muchos trabajos actuales.

\section{Referencias bibliográficas}

Abadía de Quant, I. (1996-1997). Léxico conservador en el español coloquial de Corrientes, área del nordeste argentino. Anuario de Lingüistica Hispánica, 12-13 (2), 467-481.

Abadía de Quant, I. (2001).Voces del habla de los nativos de la capital de la provincia de Corrientes. Revista Nordeste, 2da Época, 17, 7-125. https://bit.ly/3cgsUXJ.

De Ramos, P. V. (2017): O quadro de estrategias de realização do objeto direto anafórico de terceira pessoa no espanhol de Obrerá- Misiones (Argentina): Resultados. En Andrade Junior, A. F.; de Bona et al. (orgs.), Anais do XVII Colóquio de Pós-Graduação e Pesquisa em Letras Neolatinas / Colóquio 
do Programa de Pós-Graduação em Letras Neolatinas da Universidade Federal do Rio de Janeiro (pp. 220228). Universidade Federal do Rio de Janeiro, Rio de Janeiro.

Diver, W. (2012). Theory. En Huffman, A. y Davis, J., Language: communication and human behavior: The linguistic essays of William Diver (pp. 445-522). Brill Publishers (original de 1995).

García, E. (1986). The case of Spanish gender. Neuphilologische Mitteilungen, 87, 165-184.

García, E. (1992). Sincronización y desfase del leísmo y laísmo. Neuphilologische Mitteilungen, 93, 235256.

García, E. (1995). Frecuencia (relativa) de uso como síntoma de estrategias etnopragmáticas. En Zimmermann, K. (ed.), Lenguas en contacto en Hispanoamérica (pp. 51-72). Madrid / Frankfurt, Iberoamericana / Vervuert.

García, E. (1997). La portée de la variabilité. Langue française, 115, 30-47.

González Sandoval, G. (2005). Las transferencias del guarani en escuelas rurales de Corrientes. Rosario, UNR.

Granda, G. de (1982). Origen y formación del leísmo en el español del Paraguay. Ensayo de un método. RFE, LXII, 3-4, 257-283.

Hopper, P. J. (1987). Emergent grammar. Berkeley Linguistics Society, 13, 139-157.

Klein-Andrew, F. (1999). Variación actual y reinterpretación histórica: le/s, la/s, lo/s en Castilla. En Serrano, M. J. (ed.), Estudios de variación sintáctica (pp. 197-217). Madrid / Frankfurt, Iberoamericana / Vervuert.

Martínez, A. (1996). Lenguaje, pensamiento y cultura: uso de "le" en la narrativa oral no estándar de Chaco y Formosa. Hispanic Linguistics, 8 (1), 94-122.

Martínez, A. (2000). Lenguaje y Cultura. Estrategias etnopragmáticas en el uso de los pronombres clíticos lo, la y le en la Argentina en zonas de contacto con lenguas aborigenes (Tesis doctoral). Holanda, Universidad de Leiden.

Martínez, A. (2008). Fronteras teóricas y sintaxis sin fronteras: Uso de clíticos átonos en español de Buenos Aires. Páginas de Guarda. Revista de lenguaje, edición y cultura escrita, 6, 12-26.

Martínez, A. (2010). Lenguas y variedades en contacto. Problemas teóricos y metodológicos. En Martínez, A. y Palacios, A. (eds.), Lenguas en contacto en Hispanoamérica, RILI, 15, 9-31.

Martínez, A. (2013). Los pronombres clíticos lo, la, le en el español de la Argentina. En Collantoni, L. y Rodríguez, C. Perspectivas teóricas y experimentales sobre el español argentino (pp. 397-416). Madrid / Frankfurt, Vervuert / Iberoamericana.

Martínez, A. (2019). El “juego" intraparadigmático: Una mirada al uso actual de los clíticos en Buenos Aires. En Stern, N., Otheguy, R., Reid, W.y Sackler, J. (eds.), Columbia School Linguistics in the 21st Century (pp. 199-216). Amsterdam / Philadelphia, John Benjamins Publishing Company. 
Mauder, E. y Martínez, A. (2019). Being polite in Argentina. En Stern, N.; Otheguy, R.; Reid, W. y Sackler, J. (eds.), Columbia School Linguistics in the 21st Century (pp. 233-258). Amsterdam / Philadelphia, John Benjamins Publishing Company.

Palacios, A. (2008). Capítulo 13: Paraguay. En Palacios, A. (coord.), El español en América. Contactos lingüísticos en Hispanoamérica (pp. 279-300). Barcelona, Ariel.

Vidal de Battini, B. (1964). El español de la Argentina. Buenos Aires, Ministerio de Educación.

Vidal de Battini, B. (1984). Cuentos y Leyendas populares de la Argentina. Buenos Aires, Ediciones Culturales Argentinas.

Yausaz, F. (2005). Estrategias de adecuación en el uso de pronombres clíticos en textos orales y escritos producidos por hablantes correntinos. Revista Estudios en Ciencias Humanas. Estudios y monografías de los Posgrados de la Facultad de Humanidades UNNE, 1-15.

*Angelita Martínez es Doctora en Letras por la Universidad de Leiden (Países Bajos) y Profesora extraordinaria en carácter de Consulta de la Universidad Nacional de La Plata. Es directora del Centro de Estudios e Investigaciones Lingüísticas (CEIL), coordinadora de la Maestría en Lingüística de la misma Universidad, Delegada Regional de la Asociación de Lingüística y Filología para la América Latina (ALFAL) y responsable de la subsede La Plata de la Cátedra UNESCO, para el mejoramiento y equidad de la Lectura y la Escritura. Es directora de la serie de libros Discutir el lenguaje y curadora del Proyecto CORdEMIA (Corpus de español de migrantes en la Argentina), ambos de la UNLP. Su producción científica está ligada, especialmente, a temas de variación lingüística y contacto de lenguas. Desde hace veinte años dirige el programa de investigación Etnopragmática y participa activamente en el núcleo de los Programas internacionales "El español de los Andes” (Universidades de Friburgo y Montreal) y "Contacto del español con otras lenguas", con base en la UAM (Madrid).

RECIBIDO: 3/11/2020

ACEPTADO: $1 / 12 / 2020$ 